\title{
TOXICIDAD DE HERBICIDAS PROMISORIOS PARA EL CONTROL DE Dieffenbachia oerstedii EN HIJOS DE BANANO ${ }^{1}$
}

\author{
Steven Brenes-Prendas ${ }^{2}$,Renán Aguero-Alvarado²
}

\section{RESUMEN}

Toxicidad de herbicidas promisorios para el control Dieffenbachia oerstedii en hijos de banano. Entre los meses de agosto a diciembre del 2006, se evaluó la toxicidad potencial y el efecto sobre el crecimiento de hijos espada de banano, bajo seis tratamientos herbicidas para el control de sainillo: testigo absoluto (T1), carfentrazone + surfactante (T2), glifosato + surfactante (T3), glifosato + carfentrazone + surfactante (T4), glifosato + sulfato de amonio + surfactante (T5) y metsulfuron metil + carfentrazone + surfactante (T6). Se utilizó un diseño irrestricto al azar con 20 repeticiones, para un total de 120 hijos. El tratamiento con metsulfuron metil afectó el crecimiento de los hijos espada, causando la muerte de algunos de ellos, mientras que el glifosato + carfentrazone + surfactante no afectó a los hijos espada y no se diferenció del testigo. Se confirma el potencial del tratamiento glifosato + carfentrazone como alternativa para el control del sainillo en plantaciones comerciales de banano, pues provee un control aceptable de la maleza con selectividad a los hijos espada del cutlivo.

Palabras clave: Hijos productivos, sainillo, carfentrazone, glifosato, metsulfuron metil.

\begin{abstract}
Toxicity of banana suckers by promisory herbicides for the control of Dieffenbachia oerstedii. Potential toxicity on banana sword suckers of next treatments was evaluated between August and December of 2006: T1) absolute check, T2) Carfentrazone + surfactant; T3) Glyphosate + surfactant; T4) Glyphosate + carfentrazone + surfactant; T5) Glyphosate + ammonium sulphate + surfactatant; T6) metsulfuron metyl + carfentrazone + surfactant. A complete randomized design was used, with 20 replications for each treatment, for a total of 120 bananas suckers. The treatment with Metsulfuron methyl + carfentrazone + surfactant affected growth of some suckers and caused death of a few of them. Glyphosate + carfentrazone + surfactant did not differ from the absolute check. The potential of the treatment with Glyphosate + carfentrazone for Dieffenbachia oerstedii control in commercial fields of banana plantations is confirmed, because it provides acceptable wweed control and no damage of young banana suckers.
\end{abstract}

Key words: Productive suckers, sainillo, carfentrazone, glyphosate, metsulfuron metyl.

\section{INTRODUCCIÓN}

El propósito de controlar las arvenses del banano es evitar los efectos negativos que éstas pueden tener sobre el sistema productivo. Existen algunas especies de arvenses que escapan a las alternativas de control que se usan actualmente y que causan dificultades a la hora de realizar las prácticas agronómicas propias del cultivo, las cuales son conocidas como arvenses recalcitrantes. El sainillo (Dieffenbachia oerstedii Schott) es una de ellas.

Recibido: 18 de julio, 2011. Aceptado: 12 de marzo, 2012. Este trabajo forma parte de la tesis de maestría del primer autor.

2 Laboratorio de Arvenses. Centro de Investigación en Protección de Cultivos (CIPROC). Universidad de Costa Rica. sbrenesp@gmail.com; ragueroster@gmail.com 
Al considerar las alternativas de control para el sainillo, se deben analizar opciones eficientes de control que a su vez no afecten al cultivo, principalmente a los hijos productivos. En banano es conocida la importancia de los hijos productivos, también conocidos como hijos espada, en la unidad productiva madrehijo-nieto; ya que estos garantizan la futura cosecha (Soto 1992). Por esta razón las prácticas agronómicas, entre ellas el control de sainillo, deben evitar cualquier daño a los hijos espada.

En la actualidad, el glifosato es el herbicida más utilizado para el control de arvenses en banano, del cual se ha investigado acerca de su efecto en los hijos espada. En estudios realizados por Agüero y Pérez (1998), se concluyó que a las dosis recomendadas por la casa comercial y en aspersiones por debajo de los 60 $\mathrm{cm}$ de altura de hijos espada, este herbicida no afectó el crecimiento, desarrollo, producción ni calidad de fruta. En estudios realizados por Rodríguez y Agüero (2004), se obtuvo que aplicaciones de glifosato sobre cortes frescos de deshijas, no afectaron el crecimiento de los hijos espada. A pesar de este comportamiento deseable en banano, el glifosato por si sólo no muestra un control eficiente del sainillo.

Entre las nuevas alternativas para el control de arvenses en el agroecosistema bananero, se encuentra el herbicida carfentrazone - etil, del grupo aril triazinona, que se caracteriza por ser absorbido rápidamente por el follaje (WSSA 2002). Al mezclarse el carfentrazone con glifosato, existe una rápida absorción de ambos herbicidas por las plantas, lo que resulta en un efecto sinergista sobre el control del sainillo y otras malezas por el glifosato; sin embargo, se desconoce si las características antes mencionadas, podrían aumentar también la absorción del glifosato en los hijos espada del banano.

El objetivo del presente estudio fue evaluar la toxicidad potencial al banano de la mezcla glifosato + carfentrazone, así como de otros tratamientos para el control de sainillo.

\section{MATERIALES Y MÉTODOS}

\section{Ubicación}

El estudio se realizó en el cable 5 de la finca Limofrut C, en Matina, provincia de Limón, en el período de agosto a diciembre del 2006. Según la clasificación de zonas de vida de Holdridge (1979), el lugar corresponde a la formación de bosque tropical húmedo, con una precipitación anual que fluctúa entre los 3500 a $4000 \mathrm{~mm}$. La temperatura anual promedio es de $25^{\circ} \mathrm{C}$, una altitud de $11 \mathrm{msnm}$ y la humedad promedio del 85\% (Herrera 1985).

\section{Metodología}

En dos secciones del cable 5 se marcaron 120 hijos espada con una altura menor a $1 \mathrm{~m}$, correspondientes a seis tratamientos, cada uno con veinte repeticiones, este número se debe a la variabilidad en crecimiento que pueden presentar los hijos de banano. La descripción de los tratamientos se detalla en el Cuadro 1. Estos fueron untados sobre los hijos a ambos lados del mismo y sobre el punto de crecimiento apical, con un mechero Microwipe (Micro Sprayers LTD), con un volumen de mezcla de $100 \mathrm{cc}$. Cada hijo recibió poco menos de $1 \mathrm{cc}$ de esa mezcla.

El diseño experimental utilizado fue un irrestricto al azar. La unidad experimental fue el hijo espada seleccionado según el criterio antes citado. Para darles seguimiento, cada hijo utilizado recibió una triple marca, a saber: una marca en la planta madre, una en el hijo y otra en una estaca al frente del hijo.

Posterior a la aplicación de los tratamientos se realizaron evaluaciones cada quince días aproximadamente. Las variables evaluadas fueron: altura del hijo, circunferencia en la base del hijo y síntomas de toxicidad. Para medir la toxicidad potencial de los hijos espada se estableció una escala (Cuadro 2).

Los tratamientos aplicados en este estudio son experimentales y no una sugerencia tácita para su aplicación en campo.

Se realizó un análisis de varianza de los datos; las medias de los tratamientos para las distintas variables se separaron con DMS protegida de Fischer, al 5\% de probabilidad.

\section{RESULTADOS Y DISCUSIÓN}

El análisis de varianza muestra que las variables de altura y circunferencia de los hijos espada no fueron diferentes en la evaluación previa al untado de los tratamientos, lo cual garantiza la homogenidad de las unidades experimentales seleccionadas (Cuadro 3). 
Cuadro 1. Tratamientos herbicidas evaluados sobre hijos espada de banano. Finca Limofrut C. Matina, Limón Costa Rica. 2006.

\begin{tabular}{cll}
\hline \# Tratamiento & Descripción de tratamiento & Dosis \\
\hline 1 & Testigo absoluto & \\
2 & Carfentrazone + surfactante & $8: 1 / 91 \% \mathrm{vpc} * / \mathrm{v}$ agua \\
3 & Glifosato + surfactante & $33: 1 / 66 \% \mathrm{vpc} / \mathrm{v}$ agua \\
4 & Glifosato + carfentrazone + surfactante & $33: 8: 1 / 58 \% \mathrm{vpc} / \mathrm{v}$ agua \\
5 & Glifosato + sulfato de amonio + surfactante & $33: 10: 1 / 56 \% \mathrm{vpc} / \mathrm{v}$ agua \\
6 & Metsulfuron metil + carfentrazone + surfactante & $19: 8: 1 / 72 \% \mathrm{vpc} / \mathrm{v}$ agua \\
\hline
\end{tabular}

* vpc / v agua: volumen de producto comercial / volumen de agua (no dura, $\mathrm{pH}$ neutro).

Cuadro 2. Escala de síntomas por toxicidad de herbicida sobre hijos espada de banano. Finca Limofrut C. Matina. Limón. Costa Rica. 2006.

\begin{tabular}{cl}
\hline Escala & Síntomas \\
\hline 0 & No hay síntomas \\
1 & Necrosis en hojuelas viejas \\
2 & Necrosis en hojuelas viejas y en nuevas \\
3 & Necrosis en hojuelas viejas y nuevas + planta torcida \\
4 & Muerte del hijo \\
\hline
\end{tabular}

$\mathrm{Al}$ evaluar el porcentaje de incremento de altura entre la primera y segunda evaluación, mediante análisis de varianza, se encontraron diferencias significativas entre tratamientos (Cuadro 4). El tratamiento con el herbicida metsulfuron metil, presentó el incremento de altura más bajo, en comparación con el testigo absoluto y los otros tratamientos. En observaciones de campo se constató que varios hijos de este tratamiento fueron afectados, presentando síntomas de grado 2 (Cuadro 2). El tratamiento de glifosato + sulfato de amonio + surfactante, presentó una condición intermedia entre el testigo absoluto y el tratamiento con metsulfuron. Los tratamientos que incluyeron al herbicida carfentrazone (T2 y T4), así como el de gifosato solo (T3) no presentaron diferencias significativas con respecto al testigo.

El porcentaje de incremento de la primera a la tercera evaluación, para el tratamiento con mesulfuron metil también presentó los valores más bajos de altura, diferencia significativa respecto al testigo (Cuadro 5).
Cuadro 3. Altura y circunferencia de hijos espada de banano antes de la aplicación de los tratamientos herbicidas. Finca Limofrut C. Limón. Costa Rica. 2006.

\begin{tabular}{ccc}
\hline Tratamientos & $\begin{array}{c}\text { Altura } \\
(\mathbf{c m})\end{array}$ & $\begin{array}{c}\text { Circunferencia } \\
(\mathbf{c m})\end{array}$ \\
\hline T1 & $44,3 \mathrm{a}$ & $22,5 \mathrm{a}$ \\
T2 & $39,5 \mathrm{a}$ & $21,3 \mathrm{a}$ \\
T3 & $47,4 \mathrm{a}$ & $20,5 \mathrm{a}$ \\
T4 & $43,5 \mathrm{a}$ & $24,4 \mathrm{a}$ \\
T5 & $53,5 \mathrm{a}$ & $23,2 \mathrm{a}$ \\
T6 & $44,4 \mathrm{a}$ & $23,4 \mathrm{a}$ \\
\hline
\end{tabular}

Medias con la misma letra en la misma columna no difieren significativamente, según DMS protegida de Fischer, $\mathrm{p}<=0,05$.

A partir de esta evaluación se perdieron algunos hijos en este tratamiento por una deshija realizada por la finca, sin embargo no se perjudicó el estudio. Se supone que hijos con síntomas severos de toxicidad indujeron a trabajadores de campo a su eliminación, a pesar de recomendaciones de antemano para evitar esto.

El tratamiento de glifosato + sulfato de amonio mantuvo el mismo comportamiento de porcentaje de incremento, pero los tratamientos de carfentrazone (T2), glifosato (T3) y glifosato + carfentrazone (T4) mostraron un comportamiento intermedio entre el testigo y el tratamiento glifosato + sulfato de amonio (Cuadro 5). Al analizar el porcentaje de incremento entre la primera y la cuarta evaluación, el comportamiento de esos tratamientos vuelve a ser igual que 
Cuadro 4. Porcentaje de aumento en la altura de hijos de banano, entre la primera ${ }^{+}$y la segunda evaluación, luego de la adición de herbicidas para el control de D. oerstedii. Finca Limofrut C. Matina, Limón, Costa Rica. 2006.

\begin{tabular}{clc}
\hline Tratamientos & Descripción de tratamientos & Ago 10 - Ago 23* \\
\hline 1 & Testigo absoluto & $24,5 \mathrm{~b}$ \\
2 & carfentrazone + surfactante & $25,1 \mathrm{~b}$ \\
3 & glifosato + surfactante & $23,6 \mathrm{~b}$ \\
4 & glifosato + carfentrazone & $22,3 \mathrm{~b}$ \\
5 & glifosato + sulfato de amonio + surfact & $17,5 \mathrm{ab}$ \\
6 & metsulfuron metil + carfentrazone + surfac & $8,6 \mathrm{a}$ \\
\hline
\end{tabular}

Medias con la misma letra no difieren significativamente, según DMS protegida de Fischer, $\mathrm{p}<=0,05$.

+ primera evaluación = un día antes de la aplicación de los tratamientos

* Separación de medias de los datos transformados a raíz de x+0,5.

Cuadro 5. Porcentaje de aumento en la altura de hijos de banano, entre la primera ${ }^{+}$y la tercera evaluación, luego de la adición de herbicidas para el control de D. oerstedii. Finca Limofrut C. Matina, Limón. Costa Rica. 2006.

\begin{tabular}{clc}
\hline Tratamientos & Descripción de tratamientos & Ago 10 - Set 13* \\
\hline 1 & Testigo absoluto & $83,9 \mathrm{c}$ \\
2 & carfentrazone + surfactante & $79,3 \mathrm{bc}$ \\
3 & glifosato + surfactante & $79,1 \mathrm{bc}$ \\
4 & glifosato + carfentrazone & $75,0 \mathrm{bc}$ \\
5 & glifosato + sulfato de amonio + surfact & $50,4 \mathrm{~b}$ \\
6 & metsulfuron metil + carfentrazone + surfac & $27.0 \mathrm{a}$ \\
\hline
\end{tabular}

Medias con la misma letra no difieren significativamente, según DMS protegida de Fischer, $\mathrm{p}<=0,05$.

${ }^{+}$primera evaluación= un día antes de la aplicación de los tratamientos.

* Separación de medias de los datos transformados a raíz x+0,5.

para la primera evaluación (Cuadro 6). Esta variación al comparar las medias se pudo deber al error experimental o de campo.

El tratamiento con metsulfuron presentó el mismo comportamiento descrito para el Cuadro 5 (Cuadro 6 y 7). Además, durante estas evaluaciones se encontraron cuatro hijos muertos en este tratamiento, y otros alcanzando síntomas de grado 3 (Cuadro 2).

El tratamiento carfentrazone + surfactante untado, no difirió del testigo a lo largo de todas las evaluaciones. El comportamiento del tratamiento de glifosato
+ carfentrazone (T4), tampoco difirió del testigo, si bien no difirió estadísticamente del tratamiento de glifosato + sulfato de amonio, las medias de este último siempre fueron menores en comparación al glifosato + carfentrazone.

El glifosato + carfentrazone untado, en los rangos de concentración estudiados, ofrece una alternativa interesante ya que además de no afectar la altura de los hijos espada, ni promover síntomas de toxicidad en hijo o madre del banano, cuenta con EPA en ese cultivo. Además, según estudios realizados por Brenes 
Cuadro 6. Porcentaje de aumento en la altura de hijos de banano, entre la primera ${ }^{+}$y la cuarte evaluación, luego de la adición de herbicidas para el control de D. oerstedii. Finca Limofrut C. Matina, Limón, Costa Rica. 2006.

\begin{tabular}{llc}
\hline Tratamientos & Descripción de tratamientos & Ago 10 - Oct 18* \\
\hline 1 & Testigo absoluto & $174,8 \mathrm{~b}$ \\
2 & carfentrazone + surfactante & $180,2 \mathrm{~b}$ \\
3 & glifosato + surfactante & $171,6 \mathrm{~b}$ \\
4 & glifosato + carfentrazone & $161,5 \mathrm{~b}$ \\
5 & glifosato + sulfato de amonio + surfact & $133,7 \mathrm{ab}$ \\
6 & metsulfuron metil + carfentrazone + surfac & $93,7 \mathrm{a}$ \\
\hline
\end{tabular}

Medias con la misma letra no difieren significativamente, según DMS protegida de Fischer, $\mathrm{p}<=0,05$. + primera evaluación $=$ un día antes de la aplicación de los tratamientos.

* Separación de medias de los datos transformados a raíz x+0,5.

Cuadro 7. Porcentaje de aumento en la altura de hijos de banano, entre la primera ${ }^{+}$y la quinta evaluación, luego de la adición de herbicidas para el control de D. oerstedii. Finca Limofrut C. Matina, Limón, Costa Rica. 2006.

\begin{tabular}{clc}
\hline Tratamientos & Descripción de tratamientos & Ago 10 - Nov 23* \\
\hline 1 & Testigo absoluto & $270,9 \mathrm{~b}$ \\
2 & carfentrazone + surfactante & $296,4 \mathrm{~b}$ \\
3 & glifosato + surfactante & $270,3 \mathrm{~b}$ \\
4 & glifosato + carfentrazone & $240,4 \mathrm{~b}$ \\
5 & glifosato + sulfato de amonio + surfact & $207,9 \mathrm{ab}$ \\
6 & Metsulfuron metil + carfentrazone + surfac & $157,7 \mathrm{a}$ \\
\hline
\end{tabular}

Medias con la misma letra no difieren significativamente, según DMS protegida de Fischer, $\mathrm{p}<=0,05$. + primera evaluación= un día antes de la aplicación de los tratamientos.

* Separación de medias de los datos transformados a raíz x+0,5.

et al. (2008), este tratamiento ofrece un control satisfactorio sobre el sainillo.

$\mathrm{Al}$ analizar el porcentaje de incremento de la circunferencia, en las diferentes evaluaciones (Cuadros $8,9,10,11)$, se observa que el tratamiento con metsulfuron metil obtuvo las circunferencias más bajas, lo cual concuerda con lo obtenido para los incrementos de altura, lo que corrobora el impacto negativo de este herbicida sobre el crecimiento del banano.

En ninguno de los tratamientos evaluados se encontraron síntomas de toxicidad típica, provocados por los herbicidas en estudio, sobre las plantas madre de banano. Debido a lo localizado de la aplicación de los tratamientos, mediante el untado de los mismo con el mechero y lo meticulosa de dicha aplicación en el campo, evitando el contacto con cualquier planta que no fuera los hijos en estudio, se estima que no hubo ninguna translocación de los herbicidas de los hijos tratados a las plantas madres de banano, por lo menos no a concentraciones que promovieran la aparición de síntomas.

El tratamiento untado de metsulfuron metil + carfentrazone + surfactante presentó los incrementos 
Cuadro 8. Porcentaje de aumento en la circunferencia de hijos de banano, entre la primera ${ }^{+}$y la segunda evaluación luego de la adición de herbicidas para el control de D. oerstedii. Finca Limofrut C. Matina, Limón. 2006.

\begin{tabular}{clc}
\hline Tratamientos & Descripción de tratamientos & Ago 10 - Ago 23 \\
\hline 1 & Testigo absoluto & $18,6 \mathrm{ab}$ \\
2 & carfentrazone + surfactante & $18,8 \mathrm{ab}$ \\
3 & glifosato + surfactante & $21,9 \mathrm{~b}$ \\
4 & glifosato + carfentrazone & $16,8 \mathrm{ab}$ \\
5 & glifosato + sulfato de amonio + surfact & $13,6 \mathrm{a}$ \\
6 & Metsulfuron metil + carfentrazone + surfac & $13,3 \mathrm{a}$ \\
\hline
\end{tabular}

Medias con la misma letra no difieren significativamente, según DMS protegida de Fischer, $\mathrm{p}<=0,05$.

+ primera evaluación $=$ un día antes de la aplicación de los tratamientos.

Cuadro 9. Porcentaje de aumento en la circunferencia de hijos de banano, entre la primera ${ }^{+}$y la tercera evaluación luego de la adición de herbicidas para el control de D. oerstedii. Finca Limofrut C. Matina, Limón. 2006.

\begin{tabular}{clc}
\hline Tratamientos & Descripción de tratamientos & Ago 10 - Set 13 \\
\hline 1 & Testigo absoluto & $49,7 \mathrm{c}$ \\
2 & carfentrazone + surfactante & $49,0 \mathrm{c}$ \\
3 & glifosato + surfactante & $44,5 \mathrm{bc}$ \\
4 & glifosato + carfentrazone & $41,6 \mathrm{abc}$ \\
5 & glifosato + sulfato de amonio + surfact & $36,6 \mathrm{ab}$ \\
6 & metsulfuron metil + carfentrazone + surfac & $30,1 \mathrm{a}$ \\
\hline
\end{tabular}

Medias con la misma letra no difieren significativamente, según DMS protegida de Fischer, $\mathrm{p}<=0,05$.

+ primera evaluación= un día antes de la aplicación de los tratamientos.

Cuadro 10. Porcentaje de aumento en la circunferencia de hijos de banano, entre la primera ${ }^{+}$y la cuarta evaluación luego de la adición de herbicidas para el control de D. oerstedii. Finca Limofrut C. Matina, Limón. 2006.

\begin{tabular}{clc}
\hline Tratamientos & Descripción de tratamientos & Ago 10 - Oct 18 \\
\hline 1 & Testigo absoluto & $81,2 \mathrm{bc}$ \\
2 & carfentrazone + surfactante & $86,4 \mathrm{c}$ \\
3 & glifosato + surfactante & $77,3 \mathrm{abc}$ \\
4 & glifosato + carfentrazone & $72,6 \mathrm{abc}$ \\
5 & glifosato + sulfato de amonio + surfact & $65,2 \mathrm{ab}$ \\
6 & metsulfuron metil + carfentrazone + surfac & $56,2 \mathrm{a}$ \\
\hline
\end{tabular}

Medias con la misma letra no difieren significativamente, según DMS protegida de Fischer, $p<=0,10$.

+ primera evaluación= un día antes de la aplicación de los tratamientos. 
Cuadro 11. Porcentaje de aumento en la circunferencia de hijos de banano, entre la primera ${ }^{+}$y la quinta evaluación luego de la adición de herbicidas para el control de D. oerstedii. Finca Limofrut C. Matina, Limón, Costa Rica. 2006.

\begin{tabular}{clc}
\hline Tratamientos & Tratamientos & Ago 10 - Nov 23 \\
\hline 1 & Testigo absoluto & $108,6 \mathrm{bc}$ \\
2 & carfentrazone + surfactante & $121,7 \mathrm{c}$ \\
3 & glifosato + surfactante & $108,2 \mathrm{abc}$ \\
4 & glifosato + carfentrazone & $98,5 \mathrm{abc}$ \\
5 & glifosato + sulfato de amonio + surfact & $91,3 \mathrm{ab}$ \\
6 & metsulfuron metil + carfentrazone + surfac & $78,3 \mathrm{a}$ \\
\hline
\end{tabular}

Medias con la misma letra no difieren significativamente, según DMS protegida de Fischer, $\mathrm{p}<=0,10$.

${ }^{+}$primera evaluación = un día antes de la aplicación de los tratamientos.

más bajos para las variables de crecimiento del cultivo evaluadas y provocó la muerte de varios hijos evaluados. Por otra parte, el metsulfuron metil no cuenta con EPA para su uso en banano, lo que limita su uso en plantaciones comerciales.

El tratamiento untado de carfentrazone + surfactante no difirió del tratamiento testigo, en las variables analizadas, lo que sugiere que, por sí mismo, a las concentraciones estudiadas, es selectivo al cultivo.

El tratamiento untado de carfentrazone + glifosato + surfactante no difirió del testigo en las variables evaluadas. Este tratamiento se constituye en la opción actual para el control químico del sainillo, ya que ofrece un control satisfactorio de esta arvense recalcitrante y no afecta el crecimiento de los hijos espada de banano.

Ninguno de los tratamientos mostró síntomas sobre las madres de banano, lo cual sugiere que los herbicidas no se transportan fuera de los hijos untados, o lo hicieron en cantidad insuficiente para provocar sintomatología visible.

Se recomienda realizar un estudio de seguimiento del tratamiento carfentrazone + glifosato + surfactante hasta cosecha, con el fin de evaluar la productividad de estos hijos.

\section{LITERATURA CITADA}

Agüero, R; Pérez, L. 1998. El uso recomendado del herbicida Ranger (glifosato a 240 G/L) no afectó el crecimiento ni el rendimiento del banano. Memorias XIII reunión ACORBAT Ecuador 98. Guayaquil. Ecuador. p. 293-305.

Brenes, S; Agüero, R; Hoffman, L. 2008. Control de sainillo (Dieffenbachia oestedii Schott) en banano. Rev. Agronomía Mesoamericana 19(2):195-208.

Herrera, W. 1985. Clima de Costa Rica: vegetación y clima de Costa Rica. Ed. UNED. Vol 2. San José. Costa Rica. 118 p.

Holdridge, L. 1979. Ecología basada en zonas de vida. IICA. San José. Costa Rica. 216 p.

Soto, M. 1992. Banano cultivo y comercialización. Litografía e imprenta LIL. Segunda edición. San José. Costa Rica. p. 30-35.

Rodríguez, A; Agüero, R. 2004. Toxicidad potencial de herbicidas postemergentes al banao sobre cortes de deshija. In Hacia un manejo racional de la flora vascular asociada al banano. Informe final. Proyecto $N^{\circ}$ 813-A1-802. Laboratorio de Malezas. Universidad de Costa Rica. 72 p.

WSSA. 2002. Herbicide Handbook. Willian H. Ahrens eds. Weed Science Society of America Publi. 352 p. 